PROCEEDINGS OF THE

AMERICAN MATHEMATICAL SOCIETY

Volume 136, Number 9, September 2008, Pages 3079-3087

S 0002-9939(08)09338-6

Article electronically published on April 30, 2008

\title{
A NOTE ON $p$-BASES OF A REGULAR AFFINE DOMAIN EXTENSION
}

\author{
TOMOAKI ONO
}

(Communicated by Bernd Ulrich)

\begin{abstract}
Let $R^{p} \subseteq R^{\prime} \subseteq R$ be a tower of commutative rings where $R$ is a regular affine domain over an algebraically closed field of prime characteristic $p$ and $R^{\prime}$ is a regular domain. Suppose $R$ has a $p$-basis $\left\{\varphi_{1}, \ldots, \varphi_{r}\right\}$ over $R^{p}$ and $\left[Q\left(R^{\prime}\right): Q\left(R^{p}\right)\right]=p^{l}(1 \leq l \leq r-1)$. For a subset $\Gamma_{r-l}$ of $R$ whose elements satisfy a certain condition on linear independence, let $M_{\Gamma_{r-l}}$ be a set of maximal ideals $\mathfrak{m}$ of $R$ such that $\Gamma_{r-l}$ is a $p$-basis of $R_{\mathfrak{m}}$ over $R_{\mathfrak{m}^{\prime}}^{\prime}$ $\left(\mathfrak{m}^{\prime}=\mathfrak{m} \cap R^{\prime}\right)$. We shall characterize this set in a geometrical aspect.
\end{abstract}

\section{INTRODUCTION}

Let $A^{p} \subseteq A^{\prime} \subseteq A$ be a tower of commutative rings of prime characteristic $p$, $\left\{\varphi_{1}, \ldots, \varphi_{r}\right\}$ a subset of $A$ and $S_{\left\{\varphi_{1}, \ldots, \varphi_{r}\right\}}$ the set of prime ideals $\mathfrak{p}$ in $A$ such that $\left\{\varphi_{1}, \ldots, \varphi_{r}\right\}$ is a $p$-basis of $A_{\mathfrak{p}}$ over $A_{\mathfrak{q}}^{\prime}\left(\mathfrak{q}=\mathfrak{p} \cap R^{\prime}\right)$. Suppose that $A$ is a Galois extension over $A^{\prime}$, where we use the notion of Galois extension in the sence of Yuan 9]. It is known that for any element $\mathfrak{p}$ of $S_{\left\{\varphi_{1}, \ldots, \varphi_{r}\right\}}$ there exists some $f \in A^{\prime}-\mathfrak{q}$ such that $\left\{\varphi_{1}, \ldots, \varphi_{r}\right\}$ is a $p$-basis of $A_{f}$ over $A_{f}^{\prime}$ (cf. the proof of Lemma 7 in 9]). Hence the Zariski open subset $\{\mathfrak{p} \in \operatorname{Spec} A \mid f \notin \mathfrak{p}\}$ of $\operatorname{Spec} A$ is contained in $S_{\left\{\varphi_{1}, \ldots, \varphi_{r}\right\}}$. This implies that $S_{\left\{\varphi_{1}, \ldots, \varphi_{r}\right\}}$ is a Zariski open set in Spec $A$. This is an easy fact for $S_{\left\{\varphi_{1}, \ldots, \varphi_{r}\right\}}$. But it seems that we do not know much information on $S_{\left\{\varphi_{1}, \ldots, \varphi_{r}\right\}}$.

Our aim is to study by a geometrical approach the subset $M_{\left\{\varphi_{1}, \ldots, \varphi_{r}\right\}}$ which consists of maximal ideals contained in $S_{\left\{\varphi_{1}, \ldots, \varphi_{r}\right\}}$ when $A$ is a regular affine domain and $A^{\prime}$ is a regular subdomain of $A$.

Throughout this paper, let $k$ always be an algebraically closed field of prime characteristic $p$, and let $R$ be the affine domain $k\left[x_{1}, \ldots, x_{n}\right] / I$ ( $I$ a prime ideal, $n \geq$ $2), R^{p}$ the affine domain $k\left[x_{1}^{p}, \ldots, x_{n}^{p}\right] / I^{(p)}\left(I^{(p)}=I \cap k\left[x_{1}^{p}, \ldots, x_{n}^{p}\right]\right)$ and $R^{\prime}$ a subring of $R$ containing $R^{p}$. Moreover $R$ and $R^{\prime}$ satisfy the following conditions:

(1) Both $R$ and $R^{\prime}$ are regular,

(2) $R$ has a $p$-basis $\left\{\varphi_{1}, \ldots, \varphi_{r}\right\}$ over $R^{p}$ where $1 \leq r=\operatorname{dim} R \leq n$,

(3) $\left[Q\left(R^{\prime}\right): Q\left(R^{p}\right)\right]=p^{l}(1 \leq l \leq r-1)$, where $Q(\star)$ is the field of fractions of a ring $\star$.

Received by the editors November 21, 2006, and, in revised form, July 27, 2007.

2000 Mathematics Subject Classification. Primary 13B99; Secondary 14A10.

Key words and phrases. Grassmannian, Kähler differential, p-basis, Zariski open set.

(C) 2008 American Mathematical Society 
By condition (1), $R$ has locally $p$-bases over $R^{\prime}$ (see the Theorem of [3] or Theorem 15.7 of [5]) so that $R$ is a Galois extension over $R^{\prime}$. Similarly $R^{\prime}$ is also over $R^{p}$.

When $R$ is a polynomial ring, a set of variables of $R$ is a $p$-basis of $R$ over $R^{p}$. Hence condition (2) is always satisfied in this case.

Let $\mathbb{A}^{n}$ be the affine $n$-space Specm $k\left[x_{1}, \ldots, x_{n}\right], X$ the non-singular subvariety Specm $R$ of $\mathbb{A}^{n}, \mathfrak{m}_{\alpha}$ the maximal ideal of $R$ corresponding to $\alpha=\left(\alpha_{1}, \ldots, \alpha_{n}\right) \in X$, $\mathfrak{m}_{\alpha}^{\prime}$ the maximal ideal $\mathfrak{m}_{\alpha} \cap R^{\prime}$ of $R^{\prime}$ and $\mathfrak{m}_{\alpha}^{(p)}$ the maximal ideal $\mathfrak{m}_{\alpha} \cap R^{p}$ of $R^{p}$. For any $\alpha \in X$, let $R_{\alpha}$ be the localization of $R$ at $\mathfrak{m}_{\alpha}, R_{\alpha}^{\prime}$ the localization of $R^{\prime}$ at $\mathfrak{m}_{\alpha}^{\prime}$ and $R_{\alpha}^{p}$ the localization of $R^{p}$ at $\mathfrak{m}_{\alpha}^{(p)}$.

Let $\Gamma_{r-l}$ be a subset $\left\{b_{1}, \ldots, b_{r-l}\right\}$ of $R$ such that the images of $d b_{1}, \ldots, d b_{r-l}$ in $\Omega_{R / R^{p}}^{1} / \mathfrak{m}_{\alpha} \Omega_{R / R^{p}}^{1}$ are linearly independent over $k$ for all $\alpha \in X$ where $d$ is the universal derivation $R \rightarrow \Omega_{R / R^{p}}$ of $R$ over $R^{p}$, and let $M_{\Gamma_{r-l}}$ be a subset of $X$ which consists of points $\alpha \in X$ such that $\Gamma_{r-l}$ is a $p$-basis of $R_{\alpha}$ over $R_{\alpha}^{\prime}$. From now on we simply denote by $\{\varphi\}$ the $p$-basis $\left\{\varphi_{1}, \ldots, \varphi_{r}\right\}$ of $R$ over $R^{p}$. First we define a map $\Psi_{\{\varphi\}}$ from $X$ to the Grassmannian $\mathbb{G}\left(l, V_{\{\varphi\}}\right)$ where $V_{\{\varphi\}}$ is the $k$ vector space $\bigoplus_{j=1}^{r} k d \varphi_{j} \subset \Omega_{R / R^{p}}^{1}$, and we shall show that this map is a morphism (Proposition 2.2). Using this morphism, we shall express $M_{\Gamma_{r-l}}$ as the complement of the projection of a closed algebraic subset in $X \times \mathbb{G}\left(l, V_{\{\varphi\}}\right)$ (Theorem 2.5). In particular, when $b_{1}, \ldots, b_{r-l}$ are given by $\sum_{j=1}^{r} c_{1 j} \varphi_{j}, \ldots, \sum_{j=1}^{r} c_{r-l j} \varphi_{j}\left(c_{i j} \in k\right)$ such that $\mathrm{rk}\left(c_{i j}\right)_{i=1 \ldots, r-l, j=1 \ldots, r}=r-l$, then $M_{\Gamma_{r-l}}$ is equal to the complement of the inverse image of some Schubert cycle of $\mathbb{G}\left(l, V_{\{\varphi\}}\right)$ (Corollary 2.6). Moreover we shall show that $R^{\prime}$ has a $p$-basis over $R^{p}$ if there exists a $p$-basis $\{\lambda\}$ of $R$ over $R^{p}$ such that the morphism $\Psi_{\{\lambda\}}: X \rightarrow \mathbb{G}\left(l, V_{\{\lambda\}}\right)$ is constant (Proposition 2.7 and Corollary 2.8), and we shall refer to what is related to the result of Ganong [1] (Corollary 2.9). Next we shall give three examples which induce the morphism $\Psi_{\{\varphi\}}$ from an affine plane to a projective line (Examples 3.3 (A), 3.4 (B) and 3.5 (C)). In particular, Examples 3.3 (A) and 3.4 (B) are the cases such that for every set of variables of an affine plane the morphism is surjective.

Our main results are based on well-known facts about $p$-bases and Kähler differentials. For general facts concerning $p$-bases of a local ring, resp. field extension, see [5], 6] or [7].

\section{MAIN RESUlts}

Let $\Omega_{R / R^{p}}^{1}$ be the module of Kähler differentials of $R$ over $R^{p}$ and $d: R \rightarrow \Omega_{R / R^{p}}^{1}$ the universal derivation of $R$ over $R^{p}$. Under the assumptions (1)-(3) in $\S 1$ we have

$$
\Omega_{R / R^{p}}^{1}=\bigoplus_{j=1}^{r} R d \varphi_{j}
$$

and $d$ is given as

$$
d a=\sum_{j=1}^{r} D_{j} a \cdot d \varphi_{j} \text { for } a \in R
$$

where $D_{j}=\partial / \partial \varphi_{j}, D_{j} \varphi_{h}=\delta_{j h}\left(h=1, \ldots, r, \delta_{j h}\right.$ is Kronecker's delta). Let $V_{\{\varphi\}}$ (or simply $V$ ) be the $k$-vector space $\bigoplus_{j=1}^{r} k d \varphi_{j} \subset \Omega_{R / R^{p}}^{1}$. It is invariant under $k$-linear transformations of $\left\{\varphi_{1}, \ldots, \varphi_{r}\right\}$ with an invertible matrix. 
We define the $\operatorname{map} d_{\varphi}^{\alpha}: R \rightarrow V_{\{\varphi\}}$ by

$$
d_{\varphi}^{\alpha} a=\sum_{j=1}^{r}\left(D_{j} a\right)(\alpha) d \varphi_{j} \text { for } a \in R
$$

where $b(\alpha)$ for $b \in R$ denotes the image of $b$ in $k=R / \mathfrak{m}_{\alpha}$. The canonical map

$$
\Omega_{R / R^{p}}^{1} \rightarrow \Omega_{R / R^{p}}^{1} / \mathfrak{m}_{\alpha} \Omega_{R / R^{p}}^{1}
$$

identifies $V_{\{\varphi\}}$ with $\Omega_{R / R^{p}}^{1} / \mathfrak{m}_{\alpha} \Omega_{R / R^{p}}^{1}$ and $d_{\varphi}^{\alpha} a$ with the canonical image of $d a$ in this module.

Since $R$ and $R^{\prime}$ have locally $p$-bases over $R^{\prime}$ and $R^{p}$, respectively, their modules of differentials are projective of ranks $r-l$ and $l$, respectively (cf. Theorem 15.7 of [5]), and one has the fundamental split-exact sequence

$$
0 \rightarrow R \otimes_{R^{\prime}} \Omega_{R^{\prime} / R^{p}}^{1} \rightarrow \Omega_{R / R^{p}}^{1} \rightarrow \Omega_{R / R^{\prime}}^{1} \rightarrow 0
$$

The above sequence induces an exact sequence of $k$-vector spaces

$$
0 \rightarrow \Omega_{R^{\prime} / R^{p}}^{1} / \mathfrak{m}_{\alpha}^{\prime} \Omega_{R^{\prime} / R^{p}}^{1} \rightarrow \Omega_{R / R^{p}}^{1} / \mathfrak{m}_{\alpha} \Omega_{R / R^{p}}^{1} \rightarrow \Omega_{R / R^{\prime}}^{1} / \mathfrak{m}_{\alpha} \Omega_{R / R^{\prime}}^{1} \rightarrow 0 .
$$

Thus the subspace $\Omega_{R^{\prime} / R^{p}}^{1} / \mathfrak{m}_{\alpha}^{\prime} \Omega_{R^{\prime} / R^{p}}^{1}$ of $\Omega_{R / R^{p}}^{1} / \mathfrak{m}_{\alpha} \Omega_{R / R^{p}}^{1}$ corresponds to a subspace of $V_{\{\varphi\}}$ of dimension $l$ which we denote by $V_{\alpha}$ and consider as a point of the Grassmannian $\mathbb{G}\left(l, V_{\{\varphi\}}\right)$.

Definition 2.1. We define a map

$$
\Psi_{\{\varphi\}}: X \rightarrow \mathbb{G}\left(l, V_{\{\varphi\}}\right)
$$

by the rule $\Psi_{\{\varphi\}}(\alpha)=V_{\alpha}$ for $\alpha \in X$, and we write

$$
Z_{\Psi_{\{\varphi\}}}:=\left\{\left(\alpha, V_{\alpha}\right) \in X \times \mathbb{G}\left(l, V_{\{\varphi\}}\right)\right\} .
$$

Clearly the map $\Psi_{\{\varphi\}}$ depends on the choice of $p$-bases of $R$ over $R^{p}$.

Proposition 2.2. The map $\Psi_{\{\varphi\}}$ is a morphism, so that $Z_{\Psi_{\{\varphi\}}}$ is a closed subvariety of $X \times \mathbb{G}\left(l, V_{\{\varphi\}}\right)$. In particular, if there exists a $p$-basis $\left\{F_{1}, \ldots, F_{l}\right\}$ of $R^{\prime}$ over $R^{p}$, then $\Psi_{\{\varphi\}}(\alpha)$ is represented by the matrix $\left(D_{j} F_{i}(\alpha)\right)_{i=1, \ldots, l, j=1, \ldots, r}$ for $\alpha \in X$.

Proof. If elements $a_{1}, \ldots, a_{l} \in R$ form a $p$-basis of $R_{\alpha}^{\prime}$ over $R_{\alpha}^{p}$, hence $\left\{d a_{1}, \ldots, d a_{l}\right\}$ is a basis of $\Omega_{R_{\alpha}^{\prime} / R_{\alpha}^{p}}$, the space $V_{\alpha}$ has the basis $\left\{d_{\varphi}^{\alpha} a_{1}, \ldots, d_{\varphi}^{\alpha} a_{l}\right\}$ and the point $V_{\alpha} \in \mathbb{G}(l, V)$ can be represented by the matrix $\left(D_{j} a_{i}(\alpha)\right)_{i=1, \ldots, l, j=1, \ldots, r}$. Since the $p$-basis is also one of $R_{\beta}^{\prime}$ over $R_{\beta}^{p}$ for points $\beta \in X$ in a neighborhood of $\alpha$, the map $\Psi_{\{\varphi\}}$ is a morphism. The last assertion follows from the above argument.

Remark 2.3. If $R$ and $R^{\prime}$ are polynomial rings over $k$ in $n$ variables and $l=1$, there always exists a $p$-basis of $R^{\prime}$ over $R^{p}$ (cf. [1], [4 for $n=2$ and [2, 8] for any $n$ ).

Similarly, if a set $\Gamma_{s}=\left\{b_{1}, \ldots, b_{s}\right\} \subset R$ is given such that the images of $d b_{1}, \ldots, d b_{s}$ in $\Omega_{R / R^{p}}^{1} / \mathfrak{m}_{\alpha} \Omega_{R / R^{p}}^{1}$ are linearly independent over $k$ for all $\alpha \in X$, then the elements $d_{\varphi}^{\alpha} b_{1}, \ldots, d_{\varphi}^{\alpha} b_{s}$ span an $s$-dimensional subspace of $V$ which is denoted by $W_{\alpha}$. Hence we can define a map

$$
\Phi_{\Gamma_{s}}: X \rightarrow \mathbb{G}\left(s, V_{\{\varphi\}}\right)
$$

by the rule $\Phi_{\Gamma_{s}}(\alpha)=W_{\alpha}$ for $\alpha \in X$ where each $W_{\alpha}$ can be represented by $\left(D_{j} b_{h}(\alpha)\right)_{h=1, \ldots, s, j=1, \ldots, r}$. Clearly $\Phi_{\Gamma_{s}}$ is also a morphism. 
Let $\Sigma_{1}\left(W_{\alpha}\right)$ be the Schubert cycle of $\mathbb{G}\left(l, V_{\{\varphi\}}\right)$ which is the subset of $l$-dimensional vector spaces that meet $W_{\alpha}$ in a subspace of dimension at least one. We write

$$
Z_{\Gamma_{s}, \Sigma_{1}}:=\bigcup_{\alpha \in X}\{\alpha\} \times \Sigma_{1}\left(W_{\alpha}\right) \subset X \times \mathbb{G}\left(l, V_{\{\varphi\}}\right) .
$$

Since $\Phi_{\Gamma_{s}}$ is a morphism, $Z_{\Gamma_{s}, \Sigma_{1}}$ is a closed algebraic subset of $X \times \mathbb{G}\left(l, V_{\{\varphi\}}\right)$.

Lemma 2.4. If $s=r-l$, then for any $\alpha \in X$ the following conditions are equivalent:

(a) $V_{\alpha} \oplus W_{\alpha}=V_{\{\varphi\}}$.

(b) $\Gamma_{s}$ is a p-basis of $R_{\alpha}$ over $R_{\alpha}^{\prime}$.

Proof. Condition (a) and the above exact sequence (**) imply that the images of $d b_{1}, \ldots, d b_{r-l}$ in $\Omega_{R / R^{\prime}}^{1} / \mathfrak{m}_{\alpha} \Omega_{R / R^{\prime}}^{1}$ form a basis of this $k$-vector space; hence $\left\{d b_{1}, \ldots, d b_{r-l}\right\}$ is a basis of $\Omega_{R_{\alpha} / R_{\alpha}^{\prime}}^{1}$ from which it follows that $\left\{b_{1}, \ldots, b_{r-l}\right\}$ is a $p$-basis of $R_{\alpha}$ over $R_{\alpha}^{\prime}$ (see (38.G) Proposition of [6]). The assertion (a) $\Rightarrow(\mathrm{b})$ follows directly from $(* *)$.

Theorem 2.5. Let $\Gamma_{r-l}$ and $Z_{\Gamma_{r-l}, \Sigma_{1}}$ be as above, $p_{1}$ the projection $X \times \mathbb{G}\left(l, V_{\{\varphi\}}\right)$ $\rightarrow X$, and $M_{\Gamma_{r-l}}$ the set of points $\alpha \in X$ such that $\Gamma_{r-l}$ is a p-basis of $R_{\alpha}$ over $R_{\alpha}^{\prime}$. Then $M_{\Gamma_{r-l}}$ is equal to the Zariski open set $X-p_{1}\left(Z_{\Psi_{\{\varphi\}}} \cap Z_{\Gamma_{r-l}, \Sigma_{1}}\right)$.

In particular, if $Z_{\Psi_{\{\varphi\}}} \cap Z_{\Gamma_{r-l}, \Sigma_{1}}=\emptyset$, then $\Gamma_{r-l}$ is a p-basis of $R$ over $R^{\prime}$.

Proof. Condition (a) in Lemma 2.4 is equivalent to $V_{\alpha} \notin \Sigma_{1}\left(W_{\alpha}\right) \subset \mathbb{G}(l, V)$. Hence Lemma 2.4 says that $M_{\Gamma_{r-l}}=X-p_{1}\left(Z_{\Psi_{\{\varphi\}}} \cap Z_{\Gamma_{r-l}, \Sigma_{1}}\right)$. Since $p_{1}$ is a closed map and $Z_{\Psi_{\{\varphi\}}} \cap Z_{\Gamma_{r-l}, \Sigma_{1}}$ is a closed subset of $X \times \mathbb{G}(l, V)$, the image $p_{1}\left(Z_{\Psi_{\{\varphi\}}} \cap Z_{\Gamma_{r-l}, \Sigma_{1}}\right)$ is closed in $X$. Hence $M_{\Gamma_{r-l}}$ is a Zariski open subset of $X$.

Now, assume that $Z_{\Psi_{\{\varphi\}}} \cap Z_{\Sigma_{1}}=\emptyset$. Then $\Gamma_{r-l}$ is a $p$-basis of $R_{\alpha}$ over $R_{\alpha}^{\prime}$ for any $\alpha \in X$ and hence a $p$-basis of $R$ over $R^{\prime}$.

Corollary 2.6. Let $\Gamma_{r-l}$ be a subset $\left\{\sum_{j=1}^{r} c_{1 j} \varphi_{j}, \ldots, \sum_{j=1}^{r} c_{r-l j} \varphi_{j}\right\}\left(c_{i j} \in k\right)$ of $R$ such that $\operatorname{rk}\left(c_{i j}\right)_{i=1, \ldots, r-l, j=1, \ldots, r}=r-l$, and $W^{r-l}$ the subspace of $V_{\{\varphi\}}$ which is spanned by $\sum_{j=1}^{r} c_{1 j} d \varphi_{j}, \ldots, \sum_{j=1}^{r} c_{r-l j} d \varphi_{j}$. Then $M_{\Gamma_{r-l}}$ is equal to the Zariski open set $X-\Psi_{\{\varphi\}}^{-1}\left(\Sigma_{1}\left(W^{r-l}\right)\right)$.

In particular, if $\operatorname{Im} \Psi_{\{\varphi\}} \cap \Sigma_{1}\left(W^{r-l}\right)=\emptyset$, then $\Gamma_{r-l}$ is a p-basis of $R$ over $R^{\prime}$.

Proof. Clearly $Z_{\Gamma_{r-l}, \Sigma_{1}}=X \times \Sigma_{1}\left(W^{r-l}\right)$. Hence $M_{\Gamma_{r-l}}=X-\Psi_{\{\varphi\}}^{-1}\left(\Sigma_{1}\left(W^{r-l}\right)\right)$.

Proposition 2.7. If there exists a p-basis $\left\{\lambda_{1}, \ldots, \lambda_{r}\right\}$ of $R$ over $R^{p}$ such that $\Psi_{\{\lambda\}}: X \rightarrow \mathbb{G}\left(l, V_{\{\lambda\}}\right)$ is constant, then $R^{\prime}$ has a p-basis $\left\{\lambda_{1}^{\prime}, \ldots, \lambda_{l}^{\prime}\right\} \subset \bigoplus_{j=1}^{r} k \lambda_{j}$ over $R^{p}$

Proof. After a suitable $k$-linear change of $\left\{\lambda_{1}, \ldots, \lambda_{r}\right\}$, we may assume that the image of $\Phi_{\{\lambda\}}$ is the vector space $U \subset V_{\{\lambda\}}$ spanned by $\left\{d \lambda_{1}, \ldots, d \lambda_{l}\right\}$. For $\alpha \in X$ choose $f \in R^{p}-\mathfrak{m}_{\alpha}^{(p)}$ such that $R_{f}^{\prime}$ has a $p$-basis $\left\{a_{1}, \ldots, a_{l}\right\}$ over $R_{f}^{p}$. In $\Omega_{R_{f} / R_{f}^{p}}^{1}$ write $d a_{i}=\sum_{j=1}^{r} \rho_{i j} d \lambda_{j}(i=1, \ldots, l)$. Then the point $U$ can be represented by the matrix $\left(\rho_{i j}(\alpha)\right)_{i=1, \ldots, l, j=1, \ldots, r}$, and since it can also be represented by $\left(\delta_{i j}\right)_{i=1, \ldots, l, j=1, \ldots, r}$ it follows that $g:=\operatorname{det}\left(\rho_{i j}\right)_{i, j=1, \ldots, l} \neq 0$. In $R_{f g}$ the matrix 
$\left(\rho_{i j}\right)_{i, j=1, \ldots, l}$ has an inverse. After multiplication by it we have relations

$$
\sum_{i=1}^{l} \sigma_{h i} d a_{i}=d \lambda_{h}+\sum_{j=l+1}^{r} \tau_{h j} d \lambda_{j}(h=1, \ldots, l) \text { with } \sigma_{h i}, \tau_{h j} \in R_{f g} .
$$

Then $\tau_{h j}(\beta)=0(h=1, \ldots, l, j=l+1, \ldots, r)$ for all maximal ideals $\mathfrak{m}_{\beta}$ of $R_{f g}$. It follows that $\tau_{h j}=0$, hence $d \lambda_{h} \in R_{f g} \otimes_{R^{\prime}} \Omega_{R^{\prime} / R^{p}}^{1}(h=1, \ldots, l)$. Since $\alpha \in X$ was arbitrary we have $d \lambda_{h} \in R \otimes_{R^{\prime}} \Omega_{R^{\prime} / R^{p}}^{1}$, and (*) implies that $d \lambda_{h}=0$ in $\Omega_{R / R^{\prime}}^{1}$, i.e. that $\lambda_{h} \in \operatorname{Ker} d=R^{\prime}(h=1, \ldots, l)$. Then $\Omega_{R^{\prime} / R^{p}}^{1}$ has the basis $\left\{d \lambda_{1}, \ldots, d \lambda_{l}\right\}$, and hence $\left\{\lambda_{1}, \ldots, \lambda_{l}\right\}$ is a $p$-basis of $R^{\prime}$ over $R^{p}$ by (38.G) Proposition of [6].

Corollary 2.8. For a polynomial ring $R$ in $n \geq 2$ variables, if there exists a set $\{\lambda\}$ of variables $\lambda_{1}, \ldots, \lambda_{n}$ of $R$ such that $\Psi_{\{\lambda\}}: \mathbb{A}^{n} \rightarrow \mathbb{G}\left(l, V_{\{\lambda\}}\right)$ is constant, then $R^{\prime}$ is a polynomial ring.

Proof. This assertion immediately follows from Proposition 2.7.

When $R^{p} \subset R^{\prime} \subset R$ is a tower of polynomial rings over $k$ in two variables, Ganong proved in [1] that there always exists a set $\{\lambda\}$ of variables $\lambda_{1}, \lambda_{2}$ of $R$ such that $R^{\prime}=k\left[\lambda_{1}^{p}, \lambda_{2}\right]$. For these variables $\lambda_{1}, \lambda_{2}, \Psi_{\{\lambda\}}: \mathbb{A}^{2} \rightarrow \mathbb{G}\left(1, V_{\{\lambda\}}\right)=\mathbb{P}^{1}$ is constant. This implies that the converse statement of the above holds for $n=2$. But we do not know whether the converse statement holds for any $n \geq 3$.

Corollary 2.9. For a polynomial ring $R$ in two variables, $R^{\prime}$ is not a polynomial ring if and only if for each set $\{\lambda\}$ of variables $\lambda_{1}, \lambda_{2}$ of $R$ the image of $\Psi_{\{\lambda\}}$ : $\mathbb{A}^{2} \rightarrow \mathbb{P}^{1}$ is equal to either $\mathbb{P}^{1}$ or $\mathbb{P}^{1}-\{p\}$ for some point $p \in \mathbb{P}^{1}$.

Proof. The "if" part is a consequence of Ganong's work. Suppose that there are two distinct points $p_{1}, p_{2} \in \mathbb{P}^{1}-\operatorname{Im} \Psi_{\{\lambda\}}$. After a suitable $k$-linear change of variables $\lambda_{1}, \lambda_{2}$ of $R$, we may assume that $p_{1}=[1,0]$ and $p_{2}=[0,1]$. Then $\lambda_{1}$, resp. $\lambda_{2}$, is a $p$-basis of $R$ over $R^{\prime}$ by Corollary 2.6. Hence $\Omega_{R / R^{\prime}}^{1}=R d \lambda_{1}=R d \lambda_{2}$ and so $d \lambda_{1}=c d \lambda_{2}$ with a unit $c \in k-\{0\}$. Therefore $\lambda_{1}-c \lambda_{2} \in \operatorname{Ker} d=R^{\prime}$ and $R^{\prime}=k\left[\lambda_{2}^{p}, \lambda_{1}-c \lambda_{2}\right]$. This asserts the "only if" part.

\section{EXAMPLES}

In this section we shall give three examples of a tower $R^{p} \subseteq R^{\prime} \subseteq R$ of rings such that $R$ is a polynomial ring over $k$ in two variables and $\left[Q\left(R^{\prime}\right): Q\left(R^{p}\right)\right]=p$. First of all we prepare the following two facts:

Lemma 3.1. Let $R=k\left[x_{1}, \ldots, x_{n}\right], R^{\prime}=R^{p}\left[F_{1}, \ldots, F_{l}\right]\left(F_{1}, \ldots, F_{l} \in R-R^{p}, 1 \leq\right.$ $l \leq n)$ and let $\mathfrak{d}^{i}\left(R / R^{\prime}\right)$ be the $i$-th Jacobian ideal of $R$ over $R^{\prime}$. Then $\mathfrak{d}^{n-l}\left(R / R^{\prime}\right)=$ $R$ if and only if $R^{\prime}$ is regular and $\left\{F_{1}, \ldots, F_{l}\right\}$ is a p-basis of $R^{\prime}$ over $R^{p}$. In particular if $l=n$ and $\mathfrak{d}^{0}\left(R / R^{\prime}\right)=R$, then $\left\{F_{1}, \ldots, F_{l}\right\}$ is a $p$-basis of $R$ over $R^{p}$. 
Proof. Consider the localization of $(*)$ at $\alpha \in \mathbb{A}^{n}$. Then by well-known facts about Fitting ideals and Theorem 15.7 of [5] we have the following:

$$
\begin{aligned}
\mathfrak{d}^{n-l}\left(R / R^{\prime}\right)=R \Leftrightarrow & \Omega_{R / R^{\prime}}^{1} \text { is locally free of rank } n-l, \text { and for each } \alpha \in \mathbb{A}^{n} \\
& \text { we can choose elements } x_{j_{1}}, \ldots, x_{j_{n-l}} \text { from the } x_{j} \text { such } \\
& \text { that }\left\{d x_{j_{1}}, \ldots, d x_{j_{n-l}}, d F_{1}, \ldots, d F_{l}\right\} \text { is a basis of } \\
& \Omega_{R_{\alpha} / R_{\alpha}^{p}}^{1} \\
\Leftrightarrow & R^{\prime} \text { is regular and }\left\{d F_{1}, \ldots, d F_{l}\right\} \text { is a basis of } \Omega_{R_{\alpha}^{\prime} / R_{\alpha}^{p}}^{1} \\
& \text { for any } \alpha \in \mathbb{A}^{n} \\
\Leftrightarrow & R^{\prime} \text { is regular and }\left\{F_{1}, \ldots, F_{l}\right\} \text { is a } p \text {-basis of } R_{\alpha}^{\prime} \text { over } R_{\alpha}^{p} \\
& \text { for any } \alpha \in \mathbb{A}^{n} .
\end{aligned}
$$

Thus the first assertion holds.

Next we consider the second assertion. Suppose $l=n$ and $\mathfrak{d}^{0}\left(R / R^{\prime}\right)=R$. Since $\left\{F_{1}, \ldots, F_{l}\right\}$ is a $p$-basis of $R^{\prime}$ over $R^{p}$ by the first assertion, we shall only show that $R=R^{\prime}$. By the first assertion $R^{\prime}$ is regular and so $R_{\alpha}$ has a $p$-basis over $R_{\alpha}^{\prime}$ (see the Theorem of [3] or Theorem 15.7 of [5]). Note that $Q(R)=Q\left(R^{\prime}\right)$. From these facts $R_{\alpha}=R_{\alpha}^{\prime}$ for any $\alpha \in \mathbb{A}^{n}$. Hence $R=\bigcap_{\alpha \in \mathbb{A}^{n}} R_{\alpha}=\bigcap_{\alpha \in \mathbb{A}^{n}} R_{\alpha}^{\prime}=R^{\prime}$.

Lemma 3.2. Let $A^{p} \subseteq A^{\prime} \subseteq A$ be a tower of factorial rings of prime charactaristic $p, \mathfrak{p}$ a height 1 prime ideal of $A$ and $\mathfrak{p}^{\prime}$ a height 1 prime ideal $\mathfrak{p} \cap A^{\prime}$ of $A^{\prime}$. Suppose all units of $A$ are contained in $A^{\prime}$. If $\mathfrak{p}=(f)$, then either $\mathfrak{p}^{\prime}=(f)$ or $\mathfrak{p}^{\prime}=\left(f^{p}\right)$.

Proof. Since $A^{\prime}$ is factorial and $\mathfrak{p}$ is the unique height 1 prime ideal of $A$ whose restriction to $A^{\prime}$ is $\mathfrak{p}^{\prime}$, there are a positive integer $d$ and a unit $g \in A$ such that $\mathfrak{p}^{\prime}=\left(f^{d} g\right)$. By the assumption $g, g^{-1} \in A^{\prime}$ so that $\mathfrak{p}^{\prime}=\left(f^{d}\right)$. Since $f^{p} \in \mathfrak{p}^{\prime}$, $f^{p}=\left(f^{d}\right)^{e} g^{\prime}$ for some positive integer $e$ and some $g^{\prime} \in A^{\prime}-\mathfrak{p}^{\prime}$. Hence $p=d e$ and $g^{\prime}=1$, and so either $d=1$ or $d=p$. If $d=1$, then $\mathfrak{p}^{\prime}=(f)$. On the other hand, if $d=p$, then $\mathfrak{p}^{\prime}=\left(f^{p}\right)$.

Example $3.3(\mathrm{~A})$. Let $R=k\left[x_{1}, x_{2}\right], F=x_{1}\left(1+x_{1}^{p} x_{2}\right)$ and $R^{\prime}=R^{p}[F]=$ $k\left[x_{1}^{p}, x_{2}^{p}, F\right]$. Then the following properties hold:

(1) $R^{\prime}$ is regular but not factorial.

(2) $\{F\}$ is a $p$-basis of $R^{\prime}$ over $R^{p}$.

(3) $R$ has no $p$-basis over $R^{\prime}$.

(4) For every set $\{\lambda\}$ of variables $\lambda_{1}, \lambda_{2}$ of $R$, the morphism $\Psi_{\{\lambda\}}: \mathbb{A}^{2} \rightarrow \mathbb{P}^{1}$ is surjective.

Since $\partial F / \partial x_{1}=1+x_{1}^{p} x_{2}$ and $\partial F / \partial x_{2}=x_{1}^{p+1}$, we have $\partial^{\prime}\left(R / R^{\prime}\right)=(1+$ $\left.x_{1}^{p} x_{2}, x_{1}^{p+1}\right)=R$. Hence, by Lemma $3.1 R$ is regular and (2) holds. Now, assume $R^{\prime}$ is factorial. Let $\mathfrak{p}$ be the ideal of $R$ which is generated by $x_{1}$, and let $\mathfrak{p}^{\prime}$ be the ideal $\mathfrak{p} \cap R^{\prime}$ of $R^{\prime}$. Then $\mathfrak{p}$, resp. $\mathfrak{p}^{\prime}$, is a height 1 prime ideal of $R$, resp. $R^{\prime}$. By Lemma $3.2, \mathfrak{p}^{\prime}$ is equal to either $\left(x_{1}\right)$ or $\left(x_{1}^{p}\right)$. Since $x_{1} \notin R^{\prime}$, it follows that $\mathfrak{p}^{\prime}=\left(x_{1}^{p}\right)$. This contradicts that $\mathfrak{p}^{\prime}$ contains $F$. Hence $R^{\prime}$ is not factorial. Therefore (1) holds.

(3) Assume $R$ has a $p$-basis $\{G\}$ over $R^{\prime}$. Then $\{F, G\}$ is a $p$-basis of $R$ over $R^{p}$. Hence, Lemma 3.1 says that the Jacobian determinant

$$
\frac{\partial F}{\partial x_{1}} \frac{\partial G}{\partial x_{2}}-\frac{\partial F}{\partial x_{2}} \frac{\partial G}{\partial x_{1}} \in k-\{0\}
$$


Writing $G$ as $\sum_{0 \leq i, j \leq p-1} a_{i, j}^{p} x_{1}^{i} x_{2}^{j}\left(a_{i, j} \in R\right)$, we have

$$
\begin{aligned}
\frac{\partial F}{\partial x_{1}} \frac{\partial G}{\partial x_{2}}-\frac{\partial F}{\partial x_{2}} \frac{\partial G}{\partial x_{1}}= & \sum_{i=0}^{p-1} \sum_{j=0}^{p-2}\left\{(j+1) a_{i j+1}^{p}+(j-i) a_{i j}^{p} x_{1}^{p}\right\} x_{1}^{i} x_{2}^{j} \\
& +\sum_{i=0}^{p-1}(p-1-i) a_{i p-1}^{p} x_{1}^{p+i} x_{2}^{p-1} .
\end{aligned}
$$

Hence we obtain the recurrence formula $a_{01}^{p} \in k-\{0\},(j+1) a_{0 j+1}^{p}+j a_{0 j}^{p} x_{1}^{p}=0$ $(1 \leq j \leq p-2)$ and $(p-1) a_{0 p-1}^{p}=0$. But the recurrence formula except for the first condition implies $a_{01}^{p}=0$. This is a contradiction. Hence $R$ has no $p$-basis over $R^{\prime}$.

(4) Suppose that there are variables $\lambda_{1}, \lambda_{2}$ of $R$ such that $\Psi_{\{\lambda\}}$ is not surjective. Then by Corollary 2.6 there exists a $p$-basis of $R$ over $R^{\prime}$. This is a contradiction to (3). Hence this assertion holds.

In general, if $R$ has no $p$-basis over $R^{\prime}$, then by Corollary 2.6 the morphism $\Psi_{\{\lambda\}}: \mathbb{A}^{2} \rightarrow \mathbb{P}^{1}$ is surjective for any set $\{\lambda\}$ of variables of $R$. From the next example we can see that the converse statement of this does not hold.

Example 3.4 (B). Let $R=k\left[x_{1}, x_{2}\right], F=x_{1}\left(1+x_{1}^{p}+x_{1}^{2 p-1} x_{2}\right)$ and $R^{\prime}=R^{p}[F]=$ $k\left[x_{1}^{p}, x_{2}^{p}, F\right]$. Then the following properties hold:

(1) $R^{\prime}$ is regular but not factorial.

(2) $\{F\}$ is a $p$-basis of $R^{\prime}$ over $R^{p}$.

(3) $R$ has a $p$-basis over $R^{\prime}$.

(4) For every set $\{\lambda\}$ of variables $\lambda_{1}, \lambda_{2}$ of $R$, the morphism $\Psi_{\{\lambda\}}: \mathbb{A}^{2} \rightarrow \mathbb{P}^{1}$ is surjective.

We can show (1) and (2) by the same argument as that in the proof of Example $3.3(\mathrm{~A})$. Since $\left\{-x_{1}+\left(1-x_{1}^{p}\right) x_{2}\right\}$ is a $p$-basis of $R$ over $R^{\prime}=R^{p}[F]$, assertion (3) holds.

(4) Suppose that there are variables $\lambda_{1}, \lambda_{2}$ of $R$ such that $\Psi_{\{\lambda\}}$ is not surjective. Then $x_{1}$ and $x_{2}$ are expressed as polynomials $f_{1}$ and $f_{2}$ in $\lambda_{1}, \lambda_{2}$, respectively, and there exists a $p$-basis of $R$ over $R^{\prime}$ by Corollary 2.6. After a suitable $k$-linear change of these variables we may assume that $\left\{\lambda_{1}\right\}$ is a $p$-basis of $R$ over $R^{\prime}$. Hence $\left\{\lambda_{1}, F\right\}$ is a $p$-basis of $R$ over $R^{p}$. According to Lemma 3.1, we have

$$
\frac{\partial \lambda_{1}}{\partial \lambda_{1}} \frac{\partial F}{\partial \lambda_{2}}-\frac{\partial \lambda_{1}}{\partial \lambda_{2}} \frac{\partial F}{\partial \lambda_{1}}=\frac{\partial F}{\partial \lambda_{2}} \in k-\{0\}
$$

Since $\partial F / \partial \lambda_{2}=\left(1+f_{1}^{p}\right) \partial f_{1} / \partial \lambda_{2}+f_{1}^{2 p} \partial f_{2} / \partial \lambda_{2}$ and $\operatorname{deg}\left(\partial f_{1} / \partial \lambda_{2}\right)<\operatorname{deg} f_{1}$, we have $f_{1}^{p} \in k$. This is a contradiction. Therefore (4) holds.

Example 3.5 (C). Let $R=k\left[x_{1}, x_{2}\right], F=x_{1}\left(1+x_{1}^{p-1} x_{2}\right)$ and $R^{\prime}=R^{p}[F]=$ $k\left[x_{1}^{p}, x_{2}^{p}, F\right]$. Then the following properties hold:

(1) $R^{\prime}$ is regular but not factorial.

(2) $\{F\}$ is a $p$-basis of $R^{\prime}$ over $R^{p}$.

(3) $R$ has a $p$-basis over $R^{\prime}$.

(4) For each set $\{\lambda\}$ of variables $\lambda_{1}, \lambda_{2}$ of $R$ the image $\operatorname{Im} \Psi_{\{\lambda\}}$ is equal to either $\mathbb{P}^{1}$ or $\mathbb{P}^{1}-\{p\}$ for some point $p \in \mathbb{P}^{1}$. Either case actually occurs. 
We can show (1) and (2) by the same argument as that in the proof of Example $3.3(\mathrm{~A})$. The morphism $\Psi_{\{x\}}: \mathbb{A}^{2} \rightarrow \mathbb{P}^{1}$ is given by $\Psi_{\{x\}}(\alpha)=\left[1, \alpha_{1}^{p}\right]$ for $\alpha=\left(\alpha_{1}, \alpha_{2}\right) \in \mathbb{A}^{2}$, and $\operatorname{Im} \Psi_{\{x\}}$ is equal to $\mathbb{P}^{1}-\{[0,1]\}$. By Corollary 2.6, $\left\{x_{2}\right\}$ is a $p$-basis of $R$ over $R^{\prime}$. Thus (3) holds. Replacing $x_{1}$ and $x_{2}$ by $x_{1}^{\prime}=x_{1}$ and $x_{2}^{\prime}=x_{2}-x_{1}^{p+1}$, the polynomial $F$ is given by $F=x_{1}^{\prime}\left(1+x_{1}^{\prime 2 p}\right)+x_{1}^{\prime p} x_{2}^{\prime}$. Then $\Psi_{\left\{x^{\prime}\right\}}: \mathbb{A}^{2} \rightarrow \mathbb{P}^{1}$ is given by $\Psi_{\left\{x^{\prime}\right\}}(\alpha)=\left[1+\alpha_{1}^{2 p}, \alpha_{1}^{p}\right]$ for $\alpha=\left(\alpha_{1}, \alpha_{2}\right) \in \mathbb{A}^{2}$, and it is surjective. Hence (4) immediately follows from these facts and Corollary 2.9, since $R$ is not a polynomial ring by (1).

In general, when $F$ is an irreducible polynomial of $k\left[x_{1}, \ldots, x_{n}\right]$ such that the subring $k\left[F, x_{1}^{p}, \ldots, x_{n}^{p}\right]$ is regular, it seems that it is not easy to know whether $k\left[F, x_{1}^{p}, \ldots, x_{n}^{p}\right]$ is a polynomial ring. As we treated the cases where $F$ is reducible in $k\left[x_{1}, x_{2}\right]$, here we give examples where $F$ is irreducible in a polynomial ring. When $F=x_{1}+x_{2}^{2}+x_{1}^{2} x_{2}^{p} \in k\left[x_{1}, x_{2}\right]$ where $p \neq 2$, the polynomial $F$ is irreducible and $k\left[F, x_{1}^{p}, x_{2}^{p}\right]$ is regular. By a similar argument as that in the proof of Example 3.4 (B) (4), we see that the morphism $\Psi_{\{\lambda\}}: \mathbb{A}^{2} \rightarrow \mathbb{P}^{1}$ is surjective for any set $\{\lambda\}$ of variables of $k\left[x_{1}, x_{2}\right]$. Hence $k\left[F, x_{1}^{p}, x_{2}^{p}\right]$ is not a polynomial ring by Corollary 2.9. On the other hand, if $F \in k\left[x_{1}, \ldots, x_{n}\right]$ is a polynomial with degree 2 and $k\left[F, x_{1}^{p}, \ldots, x_{n}^{p}\right]$ is regular where $p \neq 2$, then $F$ is irreducible and $k\left[F, x_{1}^{p}, \ldots, x_{n}^{p}\right]$ is a polynomial ring. We can show jthis as follows. After a suitable $k$-linear change of variables $x_{1}, \ldots, x_{n}$ of $k\left[x_{1}, \ldots, x_{n}\right]$, we may assume that $F$ is written as $c+$ $\sum_{i=1}^{n}\left(c_{i} x_{i}+c_{i i} x_{i}^{2}\right)$ where $c, c_{i}, c_{i i} \in k$. Since $k\left[F, x_{1}^{p}, \ldots, x_{n}^{p}\right]$ is regular and $\{F\}$ is $p$-independent over $R^{p}$, the equations $\partial F / \partial x_{1}=0, \ldots, \partial F / \partial x_{n}=0$ have no common zero by Lemma 3.1. This implies that $c_{1} \neq 0$ and $c_{11}=0$ after a suitable reodering of the variables. Hence $\left\{F, x_{2}, \ldots, x_{n}\right\}$ is a set of variables of $k\left[x_{1}, \ldots, x_{n}\right]$, and so $k\left[F, x_{1}^{p}, \ldots, x_{n}^{p}\right]=k\left[F, x_{2}^{p}, \ldots, x_{n}^{p}\right]$. Thus the assertion holds.

Remark 3.6. The following assertion immediately follows from the last assertion and Corollary 4.2 of $[8]$ :

Let $R$ be the polynomial ring $k\left[x_{1}, \ldots, x_{n}\right](n \geq 3)$, and let $R^{\prime}$ be the polynomial ring $k\left[y_{1}, \ldots, y_{n}\right]$ which is a subring of $R$ containing $R^{p}$. Suppose $p \neq 2$ and $\left[Q\left(R^{\prime}\right): Q\left(R^{p}\right)\right]=p^{2}$. If $R^{\prime}$ contains a polynomial $F$ with degree 2 in $x_{1}, \ldots, x_{n}$ such that $R^{p}[F]$ is regular, then $R^{\prime}$ has a p-basis over $R^{p}$.

\section{ACKNOWLEDGEMENT}

The author would like to express his hearty thanks to the referee for many useful suggestions that improved this paper.

\section{REFERENCES}

1. R. Ganong, Plane Frobenius sandwiches, Proc. Amer. Math. Soc. 84 (1982), 474-478. MR643732 (84h:13026)

2. N. Kanai and T. Ono, The module of differentials and p-bases of a ring extension, Comm. Alg. 28 (2000), 5369-5382. MR1785507 (2001i:13035)

3. T. Kimura and H. Niitsuma, On Kunz's conjecture, J. Math. Soc. Japan 34 (1982), 371-378. MR651278 (83h:13030)

4. 1 A note on p-basis of polynomial ring in two variables, SUT J. Math. 25 (1989), 33-38. MR1049600 (91a:13002)

5. E. Kunz, Kähler Differentials, Vieweg Advanced Lectures in Math., Vieweg \& Sohn, Braunschweig, 1986. MR864975 (88e:14025)

6. H. Matsumura, Commutative Algebra, 2nd ed., Benjamin, New York, 1980. MR575344 (82i:13003) 
7. , Commutative Ring Theory, Cambridge University Press, Cambridge, 1986. MR879273 (88h:13001)

8. T. Ono, A note on p-bases of rings, Proc. Amer. Math. Soc. 128 (2000), 353-360. MR 1623048 (2000c:13013)

9. S. Yuan, Inseparable Galois theory of exponent one, Trans. Amer. Math. Soc. 149 (1970), 163-170. MR0257063(41:1717)

Tokyo Metropolitan College of Industrial Technology, 8-17-1, Minami-senju, ARAKAWA-KU, TOKYO 116-0003, JAPAN

E-mail address: tono@kouku-k.ac.jp 